ARTICLE

Received 11 Sep 2014 | Accepted 16 Jul 2015 | Published 8 Sep $2015 \quad$ DOI: 10.1038/ncomms9094

\title{
In vivo capture and label-free detection of early metastatic cells
}

Samira M. Azarin', Ji Yi², Robert M. Gower ${ }^{3}$, Brian A. Aguado², Megan E. Sullivan ${ }^{4}$, Ashley G. Goodman ${ }^{5}$, Eric J. Jiang ${ }^{5}$, Shreyas S. Rao ${ }^{5}$, Yinying Ren ${ }^{5}$, Susan L. Tucker ${ }^{6}$, Vadim Backman ${ }^{2,7,8}$, Jacqueline S. Jeruss ${ }^{9,10} \&$ Lonnie D. Shea $5,11,12,13$

Breast cancer is a leading cause of death for women, with mortality resulting from metastasis. Metastases are often detected once tumour cells affect the function of solid organs, with a high disease burden limiting effective treatment. Here we report a method for the early detection of metastasis using an implanted scaffold to recruit and capture metastatic cells in vivo, which achieves high cell densities and reduces the tumour burden within solid organs 10-fold. Recruitment is associated with infiltration of immune cells, which include $\mathrm{Gr}^{\text {hi }} \mathrm{CD} 11 \mathrm{~b}{ }^{+}$cells. We identify metastatic cells in the scaffold through a label-free detection system using inverse spectroscopic optical coherence tomography, which identifies changes to nanoscale tissue architecture associated with the presence of tumour cells. For patients at risk of recurrence, scaffold implantation following completion of primary therapy has the potential to identify metastatic disease at the earliest stage, enabling initiation of therapy while the disease burden is low.

\footnotetext{
${ }^{1}$ Department of Chemical Engineering and Materials Science, University of Minnesota, Minneapolis, Minnesota 55455, USA. ${ }^{2}$ Department of Biomedical Engineering, Northwestern University, Evanston, Illinois 60208, USA. ${ }^{3}$ Department of Chemical Engineering, University of South Carolina, Columbia, South Carolina 29208, USA. ${ }^{4}$ Department of Pathology, Northwestern University Feinberg School of Medicine, Chicago, Illinois 60611, USA. ${ }^{5}$ Department of Chemical and Biological Engineering, Northwestern University, Evanston, Illinois 60208, USA. ${ }^{6}$ Department of Bioinformatics and Computational Biology, The University of Texas MD Anderson Cancer Center, Houston, Texas, 77030, USA. ${ }^{7}$ Chemistry of Life Processes Institute (CLP), Northwestern University, Evanston, Illinois 60208, USA.

${ }^{8}$ The Robert H. Lurie Comprehensive Cancer Center of Northwestern University, Chicago, Illinois 60611, USA. ${ }^{9}$ Department of Surgery, University of Michigan, Ann Arbor, Michigan 48105, USA. ${ }^{10}$ Department of Obstetrics and Gynecology, Northwestern University, Chicago, Illinois 60611, USA. ${ }^{11}$ Institute for BioNanotechnology in Medicine (IBNAM), Northwestern University, Chicago, Illinois 60611, USA. ${ }^{12}$ Department of Biomedical Engineering, University of Michigan, Ann Arbor, Michigan 48105, USA. ${ }^{13}$ Department of Chemical Engineering, University of Michigan, Ann Arbor, Michigan 48105, USA. Correspondence and requests for materials should be addressed to V.B. (email: v-backman@northwestern.edu) or to J.S.J. (email: jjeruss@umich.edu) or to L.D.S.(email: Idshea@umich.edu).
} 
T he discovery of metastatic spread of a primary tumour is often associated with poor prognosis, owing to the fact that metastases typically go undetected until the function of one or more organs have been affected. Identification of metastasis before significant organ invasion would enable interventional strategies to halt disease progression while the disease burden is still low ${ }^{1}$. Much attention has been focused on screening for the presence of circulating tumour cells (CTCs) as a measure of metastasis. CTCs are present at low numbers in the blood and technologies such as microfluidic devices have been designed to capture and quantify the number of CTCs from patient blood samples ${ }^{2,3}$. Recently, a system that also enables expansion of CTCs following capture has been developed ${ }^{4}$. These technologies provide opportunities for studying the biology of CTCs, development of biomarkers, disease monitoring and personalized medicine strategies. However, CTCs can remain in the circulation for long periods of time before homing to and colonizing a metastatic site, with some tumour cells being shed at early points during tumour progression $^{5,6}$. Thus, we sought to develop a method for capturing and detecting cells that have extravasated and colonized a site, which are steps in the metastatic cascade downstream from the circulation of tumour cells.

Paget's seed-and-soil hypothesis, developed a century ago, proposed that dissemination of cancer cells to specific sites in the body, such as lung or liver, is not random but rather is due to the receptive microenvironments at those sites ${ }^{7}$. More recently, studies have shown that before colonization of a metastatic site, a 'pre-metastatic niche' is established by VEGFR1 + bone marrowderived hematopoietic progenitor cells ${ }^{8}$. These cells create a tumour-supportive microenvironment comprised of several cell types, including hematopoietic and endothelial progenitor cells and immune cells that condition the environment with matrix proteins, cytokines and chemokines to facilitate migration, invasion, proliferation and angiogenesis at the metastatic site ${ }^{9-11}$. Immune cells, in particular, play critical roles in homing and colonization of the metastatic site. Macrophages facilitate extravasation of metastatic cells as they begin the process of colonization ${ }^{12}$. Myeloid-derived suppressor cells ${ }^{13-15}$ and inflammatory monocytes ${ }^{16}$ have also been associated with metastatic sites, and neutrophils have been shown to facilitate transendothelial migration of tumour cells. Importantly, the existence of the pre-metastatic niche indicates that a site could be engineered to recapitulate the microenvironment of the niche in vivo.

In this report, we develop a biomaterial implant to recruit and capture metastatic cells, combined with an imaging system using inverse spectroscopic optical coherence tomography (ISOCT) for label-free detection of cancer cells at the implant, that together constitute a system to detect early metastases. The implants are microporous scaffolds composed of poly(lactide-co-glycolide)
(PLG), a material that is FDA approved for a variety of applications. We also investigate if the capture of extravasating metastatic cells can reduce colonization of solid organs and consequently tumour burden, which could have therapeutic implications. In addition, modulation of the local immune environment may be a versatile approach for recruiting tumour cells that is distinct from strategies that mimic the microenvironment of a target organ, such as bone $e^{17,18}$. To this end, the immune response at the implant, which consists of numerous cell types such as macrophages and neutrophils, is hypothesized to mediate recruitment of tumour cells $8,10,12,16,19,20$, and this mechanism of recruitment is investigated through localized delivery of the chemokine CCL22 and transplantation of myeloid-derived suppressor cells. Recruited tumour-associated myeloid-derived suppressor cells, in part, contribute to the formation a pre-metastatic niche, which supports a permissive environment for the capture of tumour cells in the scaffold. This approach for immune cell-mediated capture and early detection of metastatic cells has the potential to be broadly applicable to many types of cancer.

\section{Results}

Scaffolds for in vivo capture of metastasizing cells. An orthotopic model of human breast cancer metastasis was employed to investigate the capture of metastatic cells at a biomaterial implant. tdTomato- and luciferase-expressing MDA-MB-231BR (231BR) cells, a highly metastatic variant of the MDA-MB-231 cell line ${ }^{21}$, were transplanted into the right mammary fat pads of female NOD/SCID-IL2R $\gamma^{-1-}$ (NSG) mice (Fig. 1c). One week after tumour inoculation, microporous PLG scaffolds (5-mm diameter, 2-mm height, Supplementary Fig. $1 \mathrm{a}-\mathrm{c}$ ) were implanted into the peritoneal fat pads, a site to which 231BR cells are not reported to colonize, yet supports the vascularization of PLG scaffolds. Bioluminescence imaging and histological analysis of peritoneal fat pads removed 28 days after tumour inoculation demonstrated the presence of tumour cells within the implanted PLG scaffold (Fig. 1a,d) and the absence of tumour cells in fat pads without scaffolds (Fig. 1b,e), indicating that the local environment generated by implantation of the scaffold enabled recruitment of metastatic cells. Primary tumour growth was not affected by either implantation of scaffolds or a mock surgery (Supplementary Fig. 2). Staining for fibronectin, a matrix protein reported to be involved in establishment of the pre-metastatic niche, indicated that fibronectin was present in scaffolds implanted in both healthy and tumour-bearing mice as early as 7 days post implantation (Supplementary Fig. 1d). Interestingly, recruitment of cells to the scaffold was not site-specific, as tumour cells were detected in scaffolds implanted in the subcutaneous tissue (Supplementary Fig. 3).
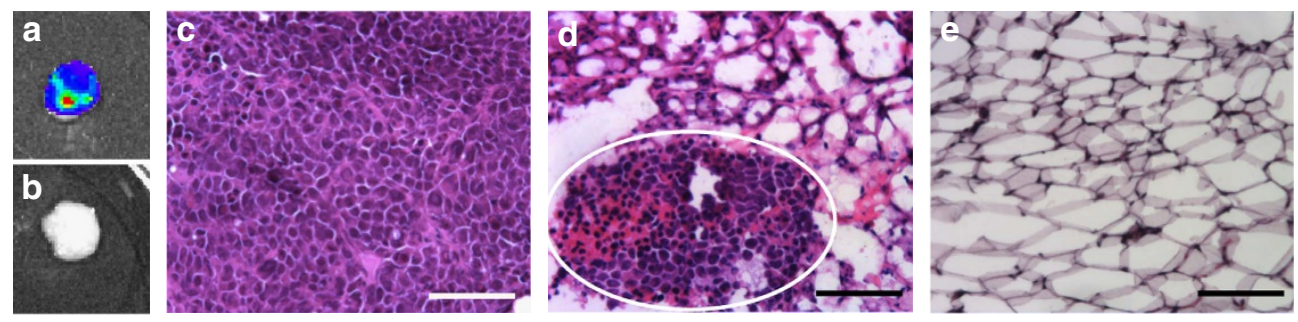

Figure 1 | PLG scaffolds recruit metastatic tumour cells. Tissues were isolated at day 28 post-tumour inoculation (21 days after scaffold implantation or mock surgery). (a,b) Bioluminescence imaging (BLI) of peritoneal fat pads receiving scaffold implants (a) or mock surgeries (b). (c-e) Hematoxylin and eosin (H\&E) staining of the primary tumour (c) a fat pad containing a scaffold (white circle indicates metastatic cluster) (d) and a fat pad without a scaffold (e). Scale bars, $100 \mu \mathrm{m}$. 
Scaffolds reduce tumour burden in solid organs. We subsequently investigated whether capturing tumour cells in scaffolds would reduce colonization of standard metastatic sites, such as the lung and liver. At 28 days post-tumour inoculation, the relative abundance of tumour cells, reported as the ratio of tdTomato-positive tumour cells to total cells, was determined. For mice that received scaffolds, the relative abundance of tumour cells in the lung was 1:5,400, compared with 1:645 for mice receiving a mock surgery (Fig. 2a, Supplementary Fig. 4). Thus, the presence of a scaffold reduced the tumour burden for the lung by $88 \pm 7 \%$ (average \pm s.e.m.). Histological analysis of lung sections confirmed a reduction in the tumour cell burden with scaffold implantation (Fig. 2b,c), with an average of $1.7 \pm 0.5$ metastatic lesions per section observed in the lungs of scaffold-bearing mice, compared with $5.5 \pm 1.7$ lesions per section in mice receiving mock surgeries. Furthermore, flow cytometric analysis of cells isolated from the liver showed detectable tumour cells in eight out of eight mice receiving mock surgeries, while mice receiving scaffold implants only exhibited detectable tumour cells in two of eight livers $(P<0.01$, Fisher's exact test).

Early detection of tumour cells in scaffold. The potential to use scaffolds for early detection of metastasis was determined by quantifying the percentage of tumour cells in intraperitoneal and subcutaneous scaffolds compared with the lung and liver at day 14 post-tumour inoculation. In a group of eight mice, most intraperitoneal scaffolds $(15 / 16)$ contained tumour cells at this time point, while none of the mice had detectable tumour cells in the lung and liver (Fig. 3a). In a separate group of mice, all subcutaneous scaffolds (10/10) contained tumour cells. The incidence of detectable metastatic disease at this early time point was lower than at day 28 post-tumour inoculation. At day 28 post-tumour inoculation in scaffold-bearing mice, the lung and liver exhibited tumour cells in 8 and 2 of the eight mice, respectively. Furthermore, for mice receiving mock surgeries instead of scaffold implants, the incidence of metastatic cells in both the lung and liver increased to eight out of eight mice. Importantly, at day 14 post inoculation, while none of the lungs and livers exhibited detectable tumour cells, both intraperitoneal and subcutaneous scaffolds had a detectable percentage of tumour cells $(0.019 \pm 0.005 \%$ for intraperitoneal scaffolds and $0.044 \pm 0.017 \%$ for subcutaneous scaffolds) (Fig. 3b, Supplementary Fig. 5). This ability to detect tumour cells in the scaffold before detection in the lungs and liver may enable the early detection of metastatic disease through imaging the scaffold.

Label-free detection of metastasis at the scaffold. ISOCT ${ }^{22}$ was applied to directly visualize the scaffold architecture and provide quantitative measurement of the ultrastructural changes induced by the cancer cells. ISOCT is a light scattering-based technique capable of non-invasive three-dimensional (3D) imaging of tissue morphology with micron-level resolution and millimeter-level penetration depth ${ }^{23,24}$. In addition, for each $3 \mathrm{D}$ resolution voxel $(15 \times 15 \times 2 \mu \mathrm{m})$ ISOCT also performs a spectroscopic analysis and quantifies the power of the spectra by a scattering model $I(\lambda) \propto \lambda^{D-4} 22 . D$ is the shape factor that physically defines the macromolecular density correlation function for a range of length scales from $\sim 40$ to $350 \mathrm{~nm}$ (ref. 25), with higher $D$ values indicating a more clumped structure. It has been demonstrated that $D$ is a ubiquitous marker of the ultrastructural alterations in the early stages of various cancer types despite their different etiologies ${ }^{26-30}$, with both neoplastic cells and the surrounding stroma exhibiting an increase in $D$ in part due to chromatin condensation and collagen remodelling, respectively ${ }^{27,30,31}$.
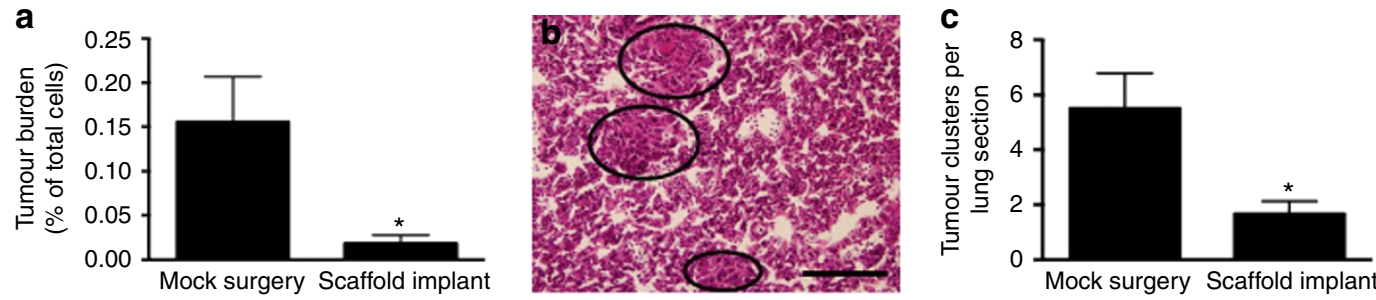

Figure 2 | Recruitment of tumour cells to scaffolds reduces tumour burden in lung. (a) Flow cytometric analysis of the percentage of tdTomato-positive tumour cells in cells isolated from lungs at day 28 post-tumour inoculation. Data shown as mean \pm s.e.m. $(n=8,2$ independent biological replicates). ${ }^{\star} P<0.01$ compared with mock surgery (Mann-Whitney test). (b) H\&E staining of lung section (black circles indicate metastatic clusters). Scale bar, $200 \mu \mathrm{m}$. (c) Histological analysis of H\&E-stained lung sections to determine the number of tumour clusters per section. Data shown as mean $\pm \mathrm{s}$.e.m. $\left(n=12,2\right.$ independent biological replicates). ${ }^{\star} P<0.05$ compared with mock surgery (Mann-Whitney test).

a

\begin{tabular}{l|c|c|c|c} 
Tissue & $\begin{array}{c}\text { Day } 14 \\
\text { incidence of } \\
\text { metastasis }\end{array}$ & $\begin{array}{c}P \text { value } \\
\text { (compared with } \\
\text { IP scaffold) }\end{array}$ & $\begin{array}{c}\text { Day } 28 \\
\text { incidence of } \\
\text { metastasis }\end{array}$ & $\begin{array}{c}P \text { value } \\
\text { (compared with } \\
\text { IP scaffold) }\end{array}$ \\
\hline Lung & 0 of 8 & $<0.0001$ & 8 of 8 & 1.0000 \\
Liver & 0 of 8 & $<0.0001$ & 2 of 8 & 0.0002 \\
IP scaffold & 15 of 16 & & 16 of 16 &
\end{tabular}

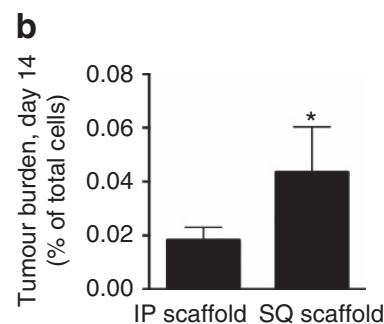

Figure 3 | Early detection of tumour cells in scaffolds. Flow cytometric analysis of tdTomato-positive tumour cells in tissues isolated from mice at day 14 and 28 post-tumour inoculation. (a) Number of mice with tumour cells detectable in each tissue in a group of 8 mice at day 14 or 28 post-tumour inoculation. Each mouse received two intraperitoneal (IP) scaffolds. $P$ values from Fisher's exact test. (b) Percentage of tdTomato-positive cells in the total cell population isolated from IP scaffolds and subcutaneous (SQ) scaffolds at day 14 post-tumour inoculation. Data shown as mean \pm s.e.m. ( $n=16$ for IP scaffold, $n=10$ for SQ scaffold, two independent biological replicates). ${ }^{\star} P<0.05$ compared with IP scaffold (Mann-Whitney test). 
a

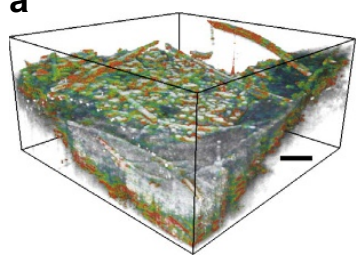

b

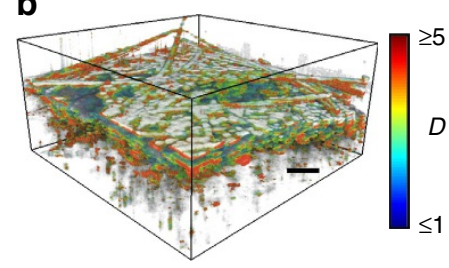

c

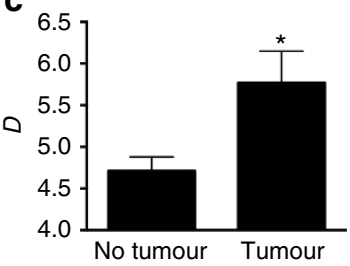

Figure 4 | Detection of tumour cells in scaffold using ISOCT. (a,b) Representative 3D maps of D generated from in situ ISOCT analysis of subcutaneous scaffolds implanted in tumour-free (a) and tumour-bearing (b) mice at day 14 post-tumour inoculation. Scale bars, $200 \mu m$. (c) Average $D$ value for subcutaneous scaffolds in tumour-free ('No Tumor') and tumour-bearing ('Tumor') mice. Data shown as mean \pm s.e.m. ( $n=6,2$ independent biological replicates). ${ }^{\star} P<0.05$ compared with tumour-free mice (Mann-Whitney test).

Given the nanoscale sensitivity of measuring $D$ and the tissuelevel imaging capability, we hypothesized that ISOCT could be an effective approach for detection of cancer cells within the scaffold.

In vitro studies were performed to demonstrate that ISOCT could capture changes in $D$ for cells and matrices, and the technique was subsequently applied to in situ imaging of scaffolds. ISOCT analysis of 231BR cell pellets confirmed that they had a higher $D$ than normal mammary epithelial cells (MCF10A) and cells isolated from lungs of tumour-free NSG mice $(3.49 \pm 0.12,2.74 \pm 0.15$ and $3.00 \pm 0.13$, respectively, Supplementary Fig. 6a). Changes to collagen remodelling by $231 \mathrm{BR}$ cells were evaluated by culturing $231 \mathrm{BR}$ cells in collagen gels for 3 days, after which cells were extracted and the gels were analysed using ISOCT. 231BR-conditioned matrices exhibited a $D$ value of $1.69 \pm 0.08$ compared with a $D$ value of $1.29 \pm 0.04$ for gels cultured with media (Supplementary Fig. 6b). With confirmation of the technique in vitro, in situ ISOCT analysis was applied to scaffolds, which demonstrated that scaffolds implanted in the subcutaneous tissue of tumour-bearing mice also had an increase in $D$ at day 14 post-tumour inoculation compared with control scaffolds in tumour-free mice (Fig. 4), with an average $D$ value of $5.77 \pm 0.38$ in tumour-bearing mice compared with $4.71 \pm 0.17$ in tumour-free mice. This increase in $D$ is consistent with the changes associated with the presence of cancerous cells and the ensuing reorganization of the extracellular matrix. These results indicate that this method can be used for label-free detection of micrometastases within the scaffold at the early stages of metastatic disease.

Immune cells contribute to tumour cell recruitment. Given the critical role of various immune cells types in establishing the pre-metastatic niche $8,10,12,16,19,20$, we hypothesized that the immune response to the scaffold was mediating recruitment of tumour cells. For analysis of the immune environment within the scaffolds, we used an immune-competent mouse model in addition to the NSG model to account for effects of both the innate and adaptive immune response. Scaffolds implanted into $\mathrm{BALB} / \mathrm{c}$ mice inoculated with $4 \mathrm{~T} 1$ mouse breast cancer cells also demonstrated metastatic cells within the scaffold, indicating that the scaffold could still achieve homing within the context of an intact immune system (Supplementary Fig. 7).

Inflammatory cells proposed to be involved in recruiting tumour cells were characterized within the peritoneal fat pads of mice in the presence and absence of a scaffold. A high density of CD45-positive leukocytes was present in histological sections of the scaffold, with no observed CD45-positive leukocytes present in fat pads of mice receiving mock surgeries (Fig. 5a,b). The ability of CD45-positive leukocytes to influence homing of tumour cells was investigated through migration assays using media conditioned by splenocytes isolated from spleens of tumour-bearing mice, as the spleen contains a large number of immune cells and has a distribution of immune cells similar to the scaffolds, with the predominant cell type being $\mathrm{Gr} 1^{\mathrm{hi}} \mathrm{CD} 11 \mathrm{~b}{ }^{+}$cells (Supplementary Fig. 8). Migration of both cell types was significantly increased in the presence of splenocyte-conditioned media relative to unconditioned media (Fig. 5c,d), with $261 \pm 35$ migrating 231BR cells per well in conditioned media compared with $137 \pm 20$ cells in unconditioned media and $521 \pm 22$ migrating $4 \mathrm{~T} 1$ cells per well in conditioned media compared with $292 \pm 23$ cells in unconditioned media. These results indicate that paracrine signalling from immune cell populations similar to those present in the scaffold can induce migration of tumour cells.

Before and after the introduction of cancer cells, we analysed the local immune environment of the implanted scaffold, which was compared with that of the lung, a common site of breast cancer metastasis. Flow cytometric analysis demonstrated that during disease progression, the most notable change in the relative distribution of immune cells was an increase in $\mathrm{Gr} 1{ }^{\text {hi }} \mathrm{CD} 11 \mathrm{~b}^{+}$cells (Fig. 5e-h, Supplementary Figs 9-11). This increase was consistent in both the NSG and BALB/c mouse models, and was found in both the lungs and scaffolds. In the lungs of the NSG mice, the percentage of $\mathrm{Gr} 1^{\text {hi }} \mathrm{CD} 11 \mathrm{~b}^{+}$cells increased from $45 \pm 2 \%$ at day 0 (no tumour) to $52 \pm 3 \%$ at day 14 post-tumour inoculation, to $84 \pm 1 \%$ at day 28 post-tumour inoculation (Fig. 5e). Following this trend, the percentage of $\mathrm{Gr} 1{ }^{\mathrm{hi}} \mathrm{CD} 11 \mathrm{~b}^{+}$cells found in the scaffolds from the NSG mice increased from $21 \pm 1 \%$ (tumour-free mice), to $35 \pm 5 \%$ at day 14 post-tumour inoculation, to $62 \pm 2 \%$ at day 28 post-tumour inoculation (Fig. $5 \mathrm{~g}$ ). In the lungs of BALB/c mice, $\mathrm{Gr} 1{ }^{\text {hi }} \mathrm{CD} 11 \mathrm{~b}{ }^{+}$cells increased from $1 \pm 0.1 \%$ at day 0 (tumourfree mice) to $57 \pm 1 \%$ at day 14 post-tumour inoculation, to $89 \pm 2 \%$ at day 28 post-tumour inoculation (Fig. $5 \mathrm{f}$ ). Likewise, within the scaffolds from the BALB/c mice, Gr1 ${ }^{\text {hi }} \mathrm{CD} 11 \mathrm{~b}^{+}$cells increased from $0.1 \pm 0.01 \%$ (no tumour) to $32 \pm 3 \%$ at day 14 post-tumour inoculation, to $66 \pm 5 \%$ at day 28 post-tumour inoculation (Fig. 5h). These results highlight the correlation between disease exposure time and the relative abundance of $\mathrm{Gr} 1^{\mathrm{hi}} \mathrm{CD} 11 \mathrm{~b}{ }^{+}$cells found in both the lungs and scaffolds of affected animals, and show that the immune environment within a metastatic organ site is similar to that of an implanted scaffold.

We subsequently investigated the hypothesis that modulation of the inflammatory microenvironment within the scaffold site would influence recruitment of metastatic cells. Lentiviral vectors were delivered from the scaffold to promote localized transgene expression for the duration of the study (Supplementary Fig. 12). The chemokine CCL22 was selected for expression as it induces migration of splenocytes harvested from NSG mice (Supplementary Fig. 13a) yet does not influence the migration of tumour cells (Supplementary Fig. 13b). Flow cytometric analysis of CCL22 scaffolds at day 7 post implantation indicated an increase in $\mathrm{Gr} 1^{\text {hi }} \mathrm{CD} 11 \mathrm{~b}{ }^{+}$cells, which have been implicated in the pre-metastatic niche $e^{10,14,15}$, from $20 \pm 2 \%$ to $29 \pm 3 \%$ in NSG 
a

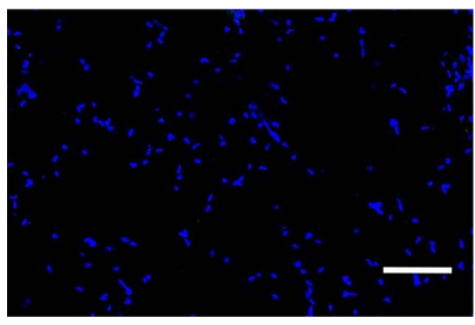

b

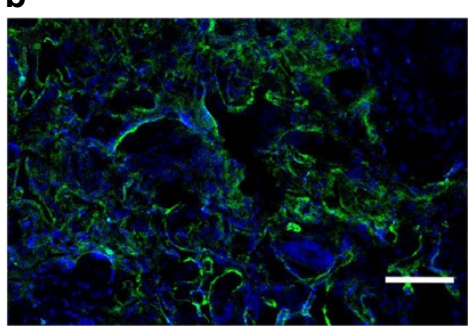

C

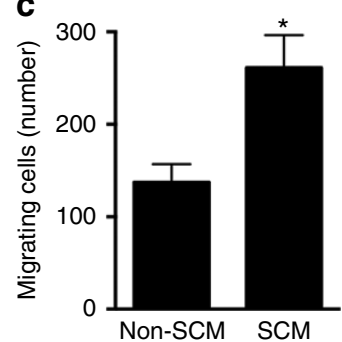

d

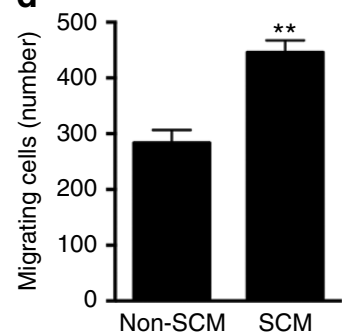

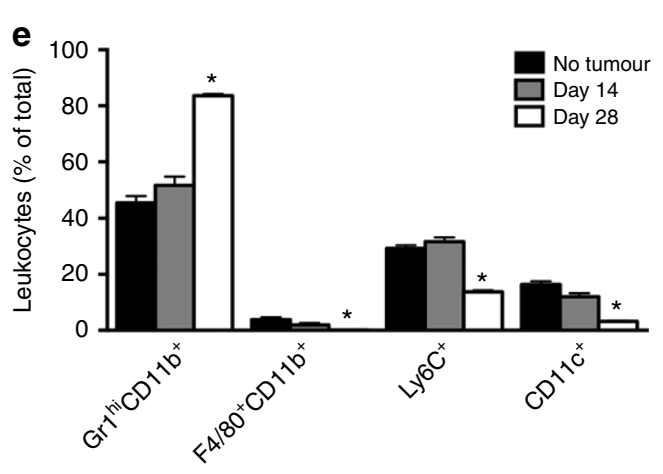
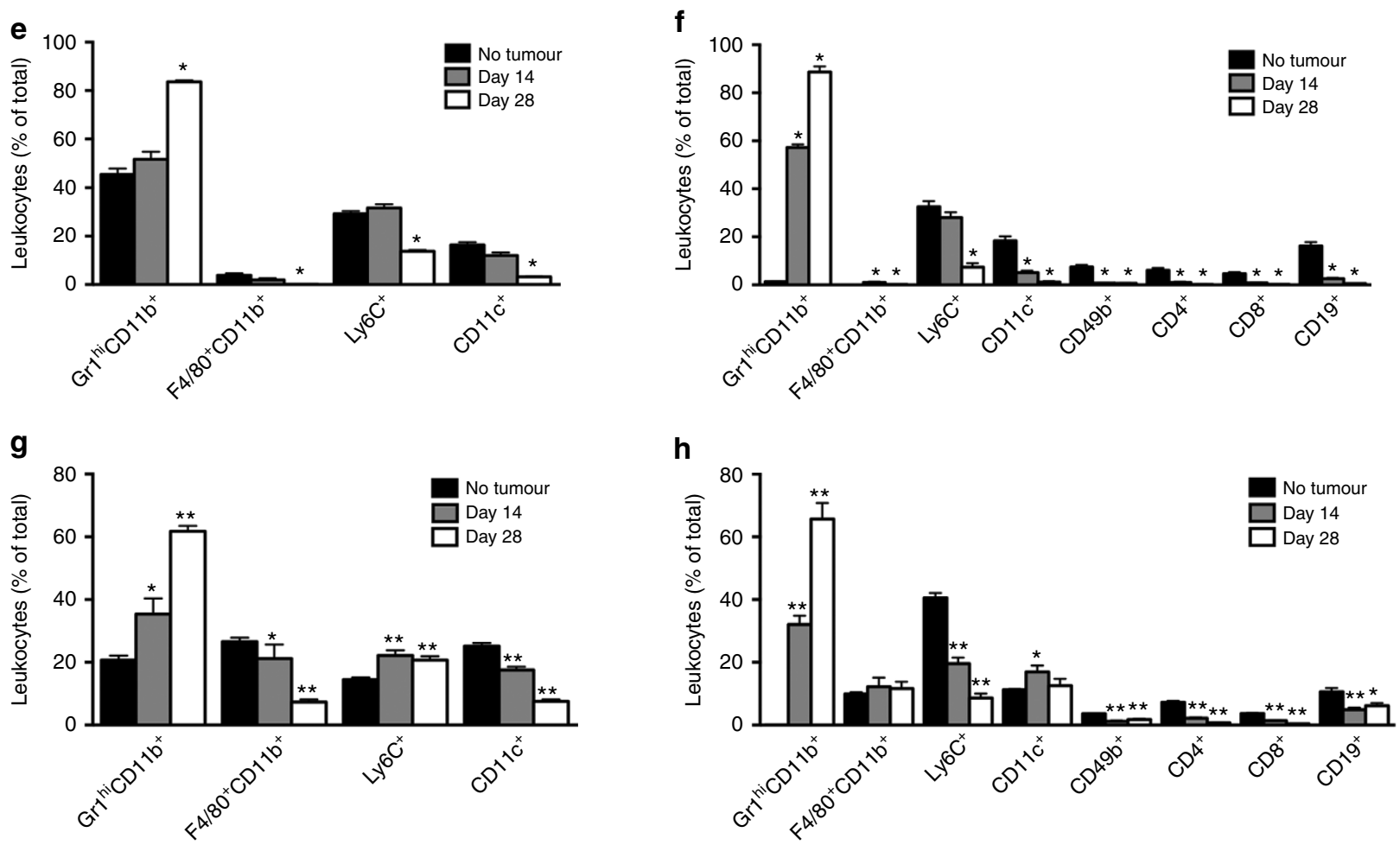

Figure 5 | Evaluation of the immune environment within scaffolds. (a,b) CD45 immunolabeling (green) at day 28 post-tumour inoculation of fat pads receiving mock surgery (a) or scaffold implant (b). Nuclei are blue. Scale bar, $100 \mu \mathrm{m}$. (c,d) Number of migrating $231 \mathrm{BR}(\mathbf{c})$ or $4 \mathrm{T1}$ (d) cells in the presence of splenocyte-conditioned media (SCM) or non-conditioned media (non-SCM). Data shown as mean \pm s.e.m. $(n=6,2$ independent biological replicates). ${ }^{\star} P<0.05$ and ${ }^{* \star} P<0.005$ compared with non-conditioned media (Mann-Whitney test). (e-h) Flow cytometric analysis of cells removed from lungs (e,f) and scaffolds $(\mathbf{g}, \mathbf{h})$ of tumour-free and tumour-bearing (day 14 and 28 ) NSG $(\mathbf{e}, \mathbf{g})$ or BALB/c (f,h) mice. The model used for mice with tumours involved the inoculation of tumour cells at day 0 , with scaffolds implanted at day 7 . The evaluation of scaffolds in tumour-free mice was performed on day 7 post implantation. Cell populations are reported as percentage of total CD45-positive leukocytes. Data shown as mean \pm s.e.m. ( $n=5$ for lungs, $n=10$ for scaffolds, 2 independent biological replicates). ${ }^{\star} P<0.05$ and ${ }^{\star \star} P<0.005$ compared with no tumour (Mann-Whitney test).

mice and from $14 \pm 1 \%$ to $19 \pm 1 \%$ in BALB/c mice (Fig. $6 a, c)$. In addition, $\mathrm{F} 4 / 80^{+} \mathrm{CD} 11 \mathrm{~b}{ }^{+}$inflammatory macrophages, which are recruited to circulating metastatic cells as they undergo extravasation and begin colonization ${ }^{12}$, increased from $28 \pm 2 \%$ to $34 \pm 2 \%$ in the NSG model and from $10 \pm 1 \%$ to $14 \pm 1 \%$ in the $\mathrm{BALB} / \mathrm{c}$ model (Fig. 6a,c). Furthermore, CCL22 expression increased the number of tdTomato-positive tumour cells present in the scaffold in both mouse models at day 7 post implantation (day 14 post-tumour inoculation), with an average of $92 \pm 16$ cells compared with an average of $23 \pm 4$ cells for $\beta$-galactosidase expression in NSG mice and an average of $62 \pm 5$ cells compared with an average of $20 \pm 2$ cells for $\beta$-galactosidase expression in BALB/c mice (Fig. 6b,d).

$A$ cell population upregulated by CCL22 delivery, $\mathrm{Gr}^{\mathrm{hi}} \mathrm{CD} 11 \mathrm{~b}^{+}$cells, was next transplanted on the scaffold to directly modulate the local immune environment and further demonstrate that the immune environment mediates metastatic cell recruitment. Gr $1{ }^{\text {hi }} \mathrm{CD} 11 \mathrm{~b}{ }^{+}$cells were selected for these studies given their established role in the pre-metastatic niche, along with migration assays showing soluble factors secreted by $\mathrm{Gr}^{\mathrm{hi}} \mathrm{CD} 11 \mathrm{~b}^{+}$cells isolated from spleens of tumour-bearing NSG mice induced migration of 231BR cells (Fig. 6e). Interestingly, the $\mathrm{Gr} 1^{\text {hi }} \mathrm{CD} 11 \mathrm{~b}^{+}$cells induced migration to a greater extent than splenocyte-conditioned media (Supplementary Fig. 14). Implantation of scaffolds seeded with $\mathrm{Gr} 1^{\mathrm{hi}} \mathrm{CD} 11 \mathrm{~b}^{+}$cells significantly increased the percentage of $\mathrm{Gr}{ }^{\mathrm{hi}} \mathrm{CD} 11 \mathrm{~b}{ }^{+}$cells in the total leukocyte population (from $20 \pm 2$ to $26 \pm 2 \%$, Fig. $6 \mathrm{f}$ ) as well as the number of tumour cells (from $29 \pm 10$ to $74 \pm 13$ cells, Fig. $6 \mathrm{~g}$ ) present per scaffold, indicating that $\mathrm{Gr} 1^{\text {hi }} \mathrm{CD} 11 \mathrm{~b}^{+}$cells contributed to recruitment of tumour cells to the scaffold.

\section{Discussion}

This study demonstrates a platform technology for the capture and label-free detection of cancer cells early in the onset of the 
a

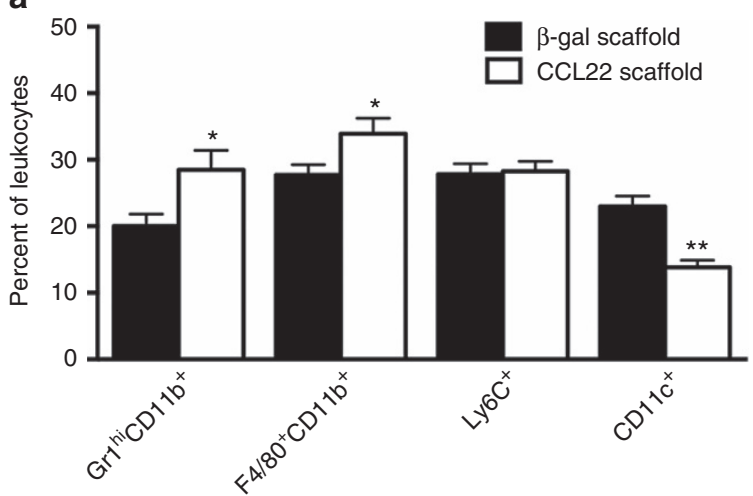

C

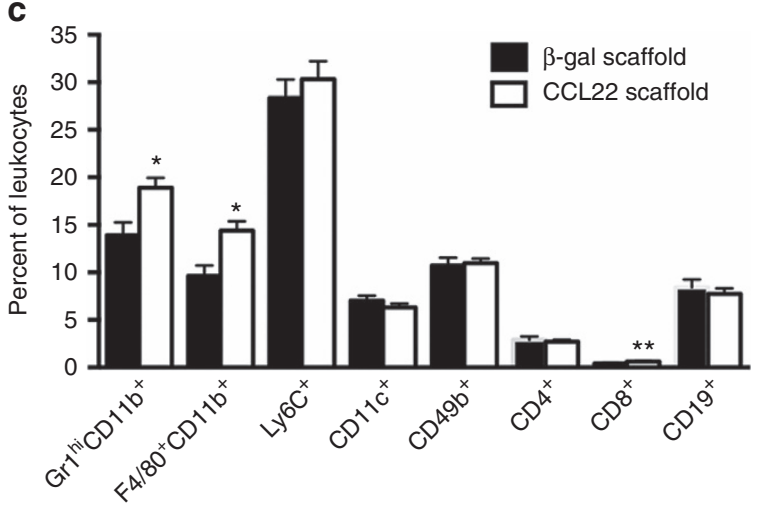

e

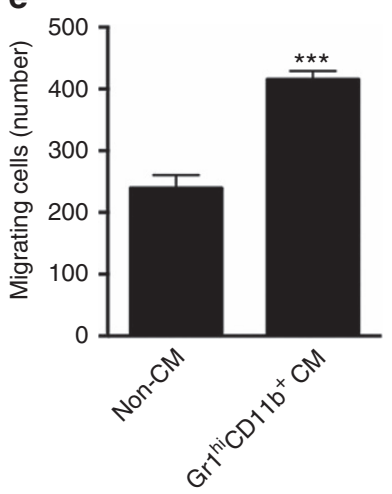

f

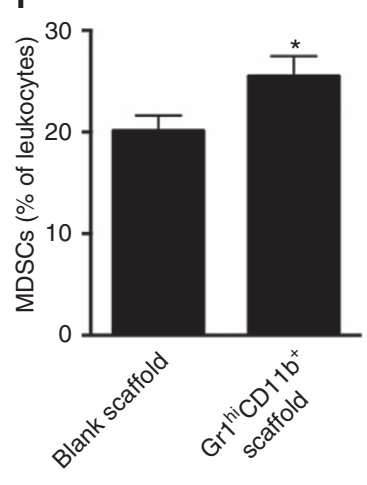

b

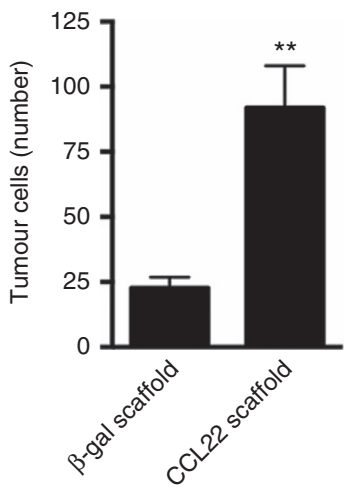

d

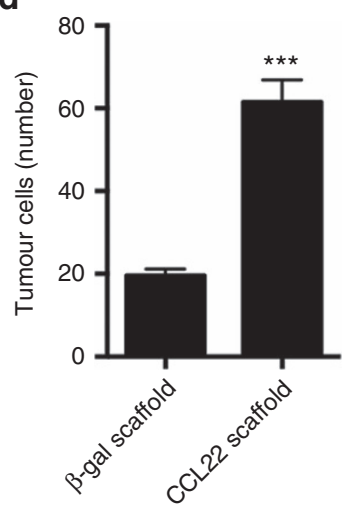

g

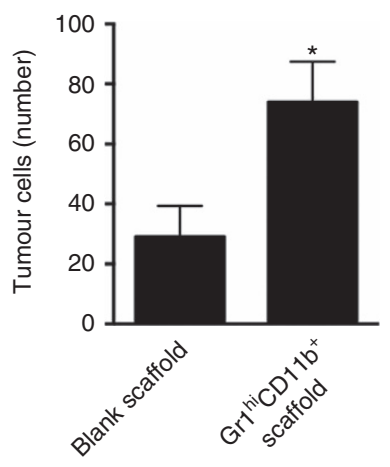

Figure 6 | Immunomodulation of the scaffold microenvironment influences recruitment of tumour cells. Flow cytometric analysis at day 14 post-tumour inoculation of cells removed from scaffolds containing a CCL22 or $\beta$-galactosidase (control) vector implanted in NSG (a,b) or BALB/c (c,d) mice. Cell populations are reported as percentage of total CD45-positive leukocytes $(\mathbf{a}, \mathbf{c})$ or total number of tumour cells in the scaffold $(\mathbf{b}, \mathbf{d})$. Data shown as mean \pm s.e.m. ( $n=10,2$ independent biological replicates). ${ }^{\star} P<0.05,{ }^{\star \star} P<0.005$ and ${ }^{\star \star \star} P<0.001$ compared with $\beta$-galactosidase scaffolds (MannWhitney test). (e) Number of migrating 231BR cells in the presence of $\mathrm{Gr} 1^{\text {hi }} \mathrm{CD} 11 \mathrm{~b}{ }^{+}$cell-conditioned media $\left(\mathrm{Gr} 1^{\text {hi }} \mathrm{CD} 11 \mathrm{~b}{ }^{+} \mathrm{CM}\right)$ or non-conditioned media (non-SCM). Data shown as mean \pm s.e.m. ( $n=6,2$ independent biological replicates). ${ }^{\star \star \star} P<0.001$ compared with non-conditioned media (Mann-Whitney test). (f,g) Flow cytometric analysis at day 14 post-tumour inoculation of cells removed from blank scaffolds or scaffolds seeded with $\mathrm{Gr}^{\text {hi }} \mathrm{CD} 11 \mathrm{~b} \mathrm{~b}^{+} \mathrm{cells}$. Cell populations are reported as the percentage of $\mathrm{Gr}^{\mathrm{hi}} \mathrm{CD} 11 \mathrm{~b}{ }^{+}$cells in the total leukocyte population (f) or total number of tumour cells in the scaffold (g). Data shown as mean \pm s.e.m. $\left(n=10,2\right.$ independent biological replicates). ${ }^{\star} P<0.05$ compared with blank scaffold (Mann-Whitney test).

metastatic process. Biomaterial scaffolds were engineered to recruit metastatic cells in vivo through modulation of the local immune environment, with detection of cancer cells based on the tissue nanoarchitecture by ISOCT. The ability of the scaffolds to reduce tumour burden in host organs that are standard sites of breast cancer metastasis suggests the potential use of the scaffold as a therapeutic tool, serving as a sink to capture CTCs. As opposed to models that employ tail vein injections to study lung colonization by directly placing large numbers of tumour cells in the bloodstream ${ }^{32}$, the orthotopic model employed in this study more closely mimics the release of metastatic cells from the primary tumour observed in the clinical setting. The ability of the scaffolds to capture a fraction of the metastatic cells early in disease progression and consequently limit colonization to other organ sites is also a distinguishing feature from purely diagnostic approaches, such as the detection of CTCs in blood samples. Furthermore, cancer cells can be retrieved from scaffolds and analysed for biomarkers unique to the metastatic setting, and targeted therapies could then be developed that are appropriately tailored to metastatic cancer cell biology. 
The approach of designing scaffolds to capture circulating cells in vivo has been used for cancer as well as regenerative medicine applications. Strategies to develop biomaterial implants that capture endothelial progenitor cells and smooth muscle progenitor cells from the bloodstream have been implemented to promote vascularization of the implant ${ }^{33-35}$. In addition, biomaterial scaffolds designed to recruit endogenous stem cells have promoted bone regeneration ${ }^{36}$ and cardiac repair ${ }^{37}$. Strategies to capture circulating cancer cells in vivo have focused on mimicking the microenvironment of a target organ, as demonstrated by the use of scaffolds that mimic the bone microenvironment for capture of breast cancer cells ${ }^{18}$ and hematopoietic cancer cells ${ }^{17}$. This technology used a broader approach of recruiting metastasizing breast cancer cells through local immune modulation, as immune cells have been shown to play critical roles in recruiting metastatic cells ${ }^{9,10,12,14-16,19}$. Given the importance of immune cells in regulating metastasis for several cancers, this approach may provide a more versatile method for capture of extravasating cancer cells in vivo. Further, the coupling of cell capture strategies with label-free imaging methods (that is, ISOCT) demonstrates the potential for using this approach as a diagnostic tool.

These scaffolds also provide an enabling tool to control the cell types and signals present at a local site to identify regulators of early events in colonization, which could lead to novel therapeutic targets for prevention of metastasis. The microenvironment within the scaffolds can be modified through cell transplantation, presentation of extracellular matrix proteins, or delivery of proteins or gene therapy vectors ${ }^{38-41}$ and represents a platform for molecularly dissecting the biological cues that underlie the recruitment of metastatic cells. Herein $\mathrm{Gr} 1{ }^{\mathrm{hi}} \mathrm{CD} 11 \mathrm{~b}{ }^{+}$cells were either recruited through chemokine expression or transplanted on the scaffold, which enhanced recruitment of extravasating tumour cells. Gr $1^{\text {hi }} \mathrm{CD} 11 \mathrm{~b}^{+}$cells accumulate in the spleen during tumour progression ${ }^{42}$, and previous reports indicate they are recruited to a pre-metastatic niche by inflammatory chemokines $^{43}$. While the increase in $\mathrm{Gr} 1^{\text {hi }} \mathrm{CD} 11 \mathrm{~b}{ }^{+}$cells in the scaffold was modest, the increase in cancer cells was substantial, which is consistent with multiple effects of $\mathrm{Gr}{ }^{\text {hi }} \mathrm{CD} 11 \mathrm{~b}^{+}$cells on tumour cells ${ }^{44}$.

Finally, scaffolds, coupled with ISOCT imaging, functioned as a sensor to detect tumour cells early in the onset of metastatic progression, which could enable therapeutic intervention while the disease burden is low. While whole-body imaging techniques do not provide the requisite sensitivity and resolution to detect a few cells as they colonize a metastatic site, optical imaging is suited for this application. Optical coherence tomography (OCT) conventionally has been used to provide microscopic reconstruction of tissue morphology with a spatial resolution on the order of several microns. Accordingly, OCT has been applied to in vivo imaging of the tumour microenvironment ${ }^{45}$. ISOCT models tissue as a medium with a continuously fluctuating macromolecular mass density ${ }^{46}$. By measuring its optical properties, the mass density correlation function can be inversely recovered at each 3D voxel of spatial resolution ${ }^{22}$. Moreover, because ISOCT uses the spectral information to measure the self-interference within a resolution-limited voxel, the length scale of sensitivity of ISOCT can be as small as $\sim 35 \mathrm{~nm}$, far beyond the resolution limit of conventional microscopy ${ }^{47-49}$. While the necessary penetration depth and large volume of a solid organ such as the lung or liver would not be well-suited for ISOCT-based detection of a small number of colonizing cells, the scaffold concentrates metastatic cells in an area beneath the skin, a site that is more readily accessible for imaging. Thus, ISOCT provides a robust approach for identifying the changes to the scaffold microenvironment on colonization by metastatic cells. Taken together, the early detection, reduced burden of metastatic disease and potential to apply targeted therapies afforded by this technology could significantly extend the time to disease progression.

\section{Methods}

Study design. The objective of the study was to use biomaterials scaffolds and optical imaging techniques to capture and detect metastasizing breast cancer cells in vivo. We hypothesized that due to the local inflammatory response following implantation, the scaffolds become infiltrated with immune cells conducive to recruiting metastatic cells, and that a light scattering-based imaging method could detect changes to the tissue ultrastructure associated with arrival of the cancer cells Animal studies were performed with at least two independent replicates of 4-8 female 10-14-week-old mice per group with random assignment. Tissues were analysed at day 14 or 28 post-tumour inoculation, with day 14 being the earliest time point for detecting metastasis and day 28 being the final time point, as the primary tumour reached the maximum allowable size. In vitro experiments had multiple samples that were independently repeated at least two times.

Tumour inoculation and volume measurement. Animal studies were performed in accordance with institutional guidelines and protocols approved by the Northwestern University Institutional Animal Care and Use Committee. Tumour inoculation was performed by injecting $2 \times 10^{6} 231 \mathrm{BR}$ (Northwestern University Developmental Therapeutics Core) or 4T1 (ATCC) cells in a volume of $50 \mu \mathrm{l}$ PBS (Life Technologies) into the number four right mammary fat pads of $10-14$-weekold female NSG or BALB/c mice. NSG mice were purchased from The Jackson Laboratory or bred in house. BALB/c mice were purchased from The Jackson Laboratory. Tumour length and width were measured weekly using digital calipers (VWR) beginning at day 14 post inoculation, and tumour volume was calculated using the formula Volume $=\left(\right.$ Width $^{2} \times$ Length $) / 2$.

Scaffold fabrication and implantation. PLG scaffolds were fabricated as previously described ${ }^{40}$. Briefly, microspheres were prepared by emulsifying a $6 \%$ solution of PLG (Lakeshore Biomaterials; 75:25 lactide:glycolide, inherent viscosity $=0.76 \mathrm{dl} \mathrm{g}^{-1}$ ) in dicholoromethane in $1 \%$ poly(vinyl alcohol). Microspheres were washed four times with deionized water to remove residual poly(vinyl alcohol) and lyophilized overnight. Next, microspheres were mixed with $250-425-\mu \mathrm{m}$ salt particles in a 30:1 ratio and pressed in a steel die at 1,500 psi. The scaffolds were then gas-foamed and salt particles were removed by washing in water for $90 \mathrm{~min}$. Scaffolds were sterilized with $70 \%$ ethanol and rinsed with water before drying. Incorporation of a viral vector was performed by incubating $1 \times 10^{9}$ viral particles in a volume of $10 \mu \mathrm{l}$ PBS with the scaffold for $8 \mathrm{~min}$. In these studies, tumours were inoculated at day 0 and scaffolds were implanted in the intraperitoneal fat pads or subcutaneous space at day 7 .

Virus production. The lentiviral vector of interest was co-transfected with the packaging vectors pRSV-Rev, pIVS-VSV-G and pMDL-GagPol ${ }^{50}$ into HEK-293T cells grown in DMEM (Sigma) containing 10\% fetal bovine serum (Life Technologies) for virus production using Lipofectamine 200 (Life Technologies). After $48 \mathrm{~h}$ the supernatant was collected. Viral particles were concentrated using PEG-it (Systems Biosciences) and resuspended in PBS. Virus titre was quantified using the qPCR Lentivirus Titration Kit (Applied Biological Materials) according to the manufacturer's instructions.

Bioluminescence imaging. Five minutes before imaging, mice were injected intraperitoneally with D-luciferin (Molecular Imaging Products; $20 \mathrm{mg} \mathrm{ml}^{-1}$ in PBS) at $150 \mathrm{mg} \mathrm{kg}^{-1}$ body weight. For whole-animal imaging, mice were anesthetized with inhaled isoflurane and in vivo luciferase expression was evaluated using the IVIS Spectrum imaging system (Caliper Life Sciences). For imaging of tissues, mice were euthanized $5 \mathrm{~min}$ after injection with $\mathrm{D}$-luciferin and tissues were retrieved and imaged using the IVIS Spectrum.

Histological analysis and immunohistochemistry. Organs and implanted scaffolds were retrieved from mice, rinsed in PBS and then immediately flash-frozen in pre-chilled isopentane. The frozen tissue was then embedded in optimal cutting temperature (OCT; Cardinal Health) compound with $30 \%$ sucrose. The lungs were sectioned at $12 \mu \mathrm{m}$, and the scaffolds and fat pads were sectioned at $14-\mu \mathrm{m}$ thick sections using a cryostat (Microm HM 525; Microm International) and stored at $-20{ }^{\circ} \mathrm{C}$ until staining. To determine the presence of tumour cells, slides were fixed with $4 \%$ paraformaldehyde (Sigma) for $10 \mathrm{~min}$ and stained with Gill III Hematoxylin (Leica) and Eosin Y (Leica). Blinded scoring of the number of tumour clusters in each tissue section was performed by a pathologist. Two sections were analysed per mouse, and in each of two separate experiments, sections from three mice per group were analysed, for a total of six animals per condition. The sections analysed were non-adjacent and separated by at least $5 \mathrm{~mm}$. For immunohistochemistry, sections were fixed for $12 \mathrm{~min}$ in $4 \%$ paraformaldehyde and stained with a polyclonal rabbit anti-CD45 primary antibody (1:100, Abcam) followed by a 
CF633 anti-rabbit secondary antibody (1:500, Sigma) or a polyclonal rabbit antifibronectin antibody (1:250, Abcam) followed by an Alexa Fluor 488 anti-rabbit secondary antibody (1:500, Life Technologies). Nuclei were labelled with Hoechst 33250 (1:2,000, Invitrogen). Samples were imaged using a Leica SP5 II laser scanning confocal microscope.

Flow cytometry. Mice were euthanized and tissues were retrieved, minced with microscissors in a $0.38 \mathrm{mg} \mathrm{ml}^{-1}$ solution of Liberase TL or TM (Roche Applied Science) in Hank's Balanced Salt Solution (HBSS; Life Technologies) and placed at $37^{\circ} \mathrm{C}$ for $20 \mathrm{~min}$. Liberase was neutralized with $0.125 \mathrm{M}$ EDTA, and cells were isolated by passing the digested tissue through a $70 \mu \mathrm{m}$ filter (BD Biosciences) and rinsing with FACS buffer, PBS containing 0.5\% Bovine Serum Albumin (BSA; Sigma) and $2 \mathrm{mM}$ EDTA (Life Technologies). Red blood cells were lysed using ACK lysing buffer (Life Technologies). For analysis of tdTomato-positive tumour cells, samples were resuspended in FACS buffer and analysed using an LSR II flow cytometer (Becton Dickinson Immunocytometry Systems, BDIS). A standard curve was established for each organ by adding defined numbers of tumour cells into cells isolated from healthy lungs and livers and measuring the number of tdTomato-positive cells via flow cytometry. From this analysis, it was determined that the threshold tumour cell density of $0.002 \%$ (five tumour cells in 250,000 total cells) was consistently and significantly above background, and thus samples were deemed to have detectable cancer cells if the tumour cell density was above $0.002 \%$. For analysis of leukocyte populations, cells were blocked with anti-CD16/32 (1:50, eBioscience) and stained for viability using fixable blue dead cell stain kit (Life Technologies). Cells were then stained with Alexa Fluor 700-conjugated anti-CD45 (30-F11, 1:125; Biolegend), Pacific Blue-conjugated anti-Gr-1 (RB6-8C5, 1:70; Biolegend), FITC-conjugated anti-Ly-6C (HK1.4, 1:100; Biolegend), PE-Cyanine7-conjugated anti-F4/80 (BM8, 1:70; Biolegend), APCconjugated anti-CD11c (N418, 1:85; eBioscience), v500-conjugated anti-CD11b (M1/70, 1:100; BD Biosciences), Pacific Blue-conjugated anti-CD19 (6D5, 1:100; Biolegend), v500-conjugated anti-CD4 (RM4-5, 1:100; BD Biosciences), FITC-conjugated anti-CD8a (53-6.7, 1:25; Biolegend) and PE-Cyanine7-conjugated anti-CD49b (DX5, 1:40; Biolegend). Samples were analysed using an LSRFortessa flow cytometer (BDIS).

\section{Splenocyte isolation for transplants and media conditioning. Spleens were} isolated from tumour-bearing mice, minced using microscissors and incubated in a $0.38 \mathrm{mg} \mathrm{ml}^{-1}$ solution of Liberase TL (Roche Applied Science) for $20 \mathrm{~min}$ at $37^{\circ} \mathrm{C}$. The minced tissue was passed through a $70 \mu \mathrm{m}$ strainer and red blood cells were lysed using ACK lysing buffer (Life Technologies). To collect $\mathrm{Gr}^{\mathrm{hi}} \mathrm{CD} 11 \mathrm{~b}{ }^{+}$cells, splenocytes were labelled with FITC-conjugated anti-Gr-1 (RB6-8C5, 1:100; Biolegend), APC-conjugated anti-Ly-6C (HK1.4, 1:50; Biolegend), and APCeFluor780-conjugated anti-CD11b (M1/70, 1:100; eBioscience) and the $\mathrm{Grl}{ }^{\text {hi }} \mathrm{CD} 11 \mathrm{~b}^{+} \mathrm{Ly}_{6 \mathrm{C}} \mathrm{C}^{-}$population was collected via FACS sorting using a $\mathrm{BD}$ FACSAria cytometer (BDIS). The remaining splenocyte population

$\left(\mathrm{Grl}{ }^{\mathrm{hi}} \mathrm{CD} 11 \mathrm{~b}^{+}\right.$cell-depleted) was also collected. For cell transplantation studies, scaffolds were rinsed with RPMI medium (Life Technologies) containing 5\% fetal bovine serum (FBS; Life Technologies) and dried on gauze. $1 \times 10^{6} \mathrm{Gr}^{\mathrm{hi}} \mathrm{CD} 11 \mathrm{~b}^{+}$ cells were seeded on each scaffold in a volume of $5 \mu \mathrm{l}$ RPMI with 5\% FBS and scaffolds were implanted into the intraperitoneal fat pads. For generation of conditioned media, splenocytes, $\mathrm{Gr} 1^{\mathrm{hi}} \mathrm{CD} 11 \mathrm{~b}{ }^{+}$cells, and $\mathrm{Gr} \mathrm{l}^{\mathrm{hi}} \mathrm{CD} 11 \mathrm{~b}{ }^{+}$ cell-depleted splenocytes were each plated at a concentration of $1 \times 10^{6}$ cells per $\mathrm{ml}$ in RPMI media. Media was conditioned for $48 \mathrm{~h}$, after which the media was sterile-filtered to remove splenocytes.

Splenocyte migration assays. Migration assays were performed in 24-well transwell chambers with $5-\mu \mathrm{m}$ pore size filters (Corning). A total of $1 \times 10^{6}$ splenocytes were placed in the top chamber in $100 \mu \mathrm{l}$ of RPMI medium containing $10 \%$ FBS. $600 \mu \mathrm{l}$ of RPMI medium containing $10 \%$ FBS with or without $100 \mathrm{ng} \mathrm{ml}^{-1}$ recombinant mouse CCL22 (Peprotech) was placed in the bottom chamber. After $1.5 \mathrm{~h}$, the number of cells that had migrated to the bottom chamber was counted using a hemocytometer.

Tumour cell migration assays. Tumour cells were serum-starved overnight in DMEM (Sigma) or RPMI (Life Technologies) containing 0.2\% FBS (Life technologies). Migration assays were performed in 24-well transwell chambers with $8-\mu \mathrm{m}$ pore size filters (Corning). Total of $5 \times 10^{4} 231 \mathrm{BR}$ or $4 \mathrm{~T} 1$ cells were placed in the top chamber in $300 \mu \mathrm{l}$ of DMEM (for CCL22 assays) or RPMI (for conditioned media assays) containing $0.2 \%$ FBS. For CCL22 assays, $750 \mu$ of DMEM containing $0.2 \%$ FBS with or without $100 \mathrm{ng} \mathrm{ml}^{-1}$ recombinant human CCL22 or SDF1 (Peprotech) was placed in the bottom chamber. For conditioned media, $750 \mu \mathrm{l}$ of RPMI, splenocyte-conditioned RPMI, Grl ${ }^{\text {hi }} \mathrm{CD} 11 \mathrm{~b}^{+}$cell-conditioned RPMI or RPMI conditioned with $\mathrm{Gr} 1{ }^{\text {hi }} \mathrm{CD} 11 \mathrm{~b}^{+}$cell-depleted splenocytes was placed in the bottom chamber (all media was supplemented with $0.2 \% \mathrm{FBS}$ ). After $24 \mathrm{~h}$, the cells on the top of the filter were removed by gently scrubbing with a cotton swab, and the cells on the bottom of the filter were stained for $60 \mathrm{~min}$ in a $0.5 \%(w / v)$ solution of crystal violet (Sigma) in 60\% PBS/40\% ethanol. The inserts were rinsed in PBS and the number of migrating cells was counted.
Conditioning collagen gels with 231BR cells. A $1.6 \mathrm{mg} \mathrm{ml}^{-1}$ collagen I solution was prepared by mixing rat tail Collagen I (BD Biosciences) with deionized water $10 \times$ PBS (Sigma, 1:10 dilution) and $1 \mathrm{~N} \mathrm{NaOH}$ (Sigma, 1:1,000 dilution) on ice. Collagen solution $(150 \mu \mathrm{l})$ was placed in each well of a 48 -well plate. Gels were incubated at $37^{\circ} \mathrm{C}$ for $1 \mathrm{~h}$, after which $1.875 \times 10^{5} 231 \mathrm{BR}$ cells in $400 \mu \mathrm{l}$ of RPMI containing $10 \%$ FBS were seeded on each gel. Control gels received $400 \mu \mathrm{l}$ of RPMI containing $10 \% \mathrm{FBS}$. After incubation at $37^{\circ} \mathrm{C}$ for 3 days, cells were extracted from the gels. The media was removed from each well and the gels were washed three times with $1 \times$ PBS (Life Technologies). A $20 \mathrm{mM} \mathrm{NH}_{4} \mathrm{OH}$ solution containing $0.5 \%(\mathrm{v} / \mathrm{v})$ Triton X-100 (Sigma) was added to each well and the chamber slide was incubated at $37^{\circ} \mathrm{C}$ for $5 \mathrm{~min}$. After removal of the $\mathrm{NH}_{4} \mathrm{OH} /$ Triton X-100 solution, the gels were washed twice with distilled water and twice with PBS. A volume of $175 \mu \mathrm{l}$ of PBS was added to each well before imaging.

Scanning electron microscopy. Structural characteristics of scaffolds were imaged with a scanning electron microscope (Hitachi s4800-II cFEG s.e.m.; Hitachi HighTechnologies Corp.). A 15-nm gold coating was applied and the microscope was operated at $2 \mathrm{kV}$

ISOCT imaging and analysis. The OCT system used a Fourier domain configuration. A supercontinuum light source (NKT photonics, SuperK versa) provided a broadband laser from $\sim 500$ to $840 \mathrm{~nm}$. The spectral range from 650 to $830 \mathrm{~nm}$ was used for OCT image construction. The laser was delivered by an optical fibre, collimated by a lens, and input into a cube beam splitter (BS; Thorlabs, CM1-BS013), by which the light was divided into a sample arm and a reference arm. The reference arm consisted of a series of glass plates for dispersion control and a mirror reflecting the light backward. The sample arm consisted of a two-dimensional scanning mirror (Thorlabs, GVSM002) and an objective lens (effective numerical aperature $=0.04$; Zeiss) to focus the light on specimens. The lateral resolution is estimated as $15 \mu \mathrm{m}$. The interfered light from the reference and sample arm at the BS was collected by another optical fibre and delivered to the spectrometer for spectral acquisition. The mirror was scanning at a frequency of $2.56 \mathrm{kHz}$, and a 3D acquisition lasted $25.6 \mathrm{~s}$ with 256 by 256 pixels in two lateral directions. The camera exposure time was $0.3 \mathrm{~ms}$. With the bandwidth, the axial resolution was estimated as $2 \mu \mathrm{m}$.

The depth structural reconstruction was created by the following steps. The recorded spectrum was first normalized by the source spectrum, and then resampled into $k$ domain with equal interval, and finally a Fourier transform was taken to recover the depth signal. $k$ is the wave number, defined by $\mathrm{k}=2 \pi / \lambda$. The calculation of $D$ value was performed by the following steps. A Gaussian window with width $k_{w}=0.36 \mu \mathrm{m}^{-1}$ was applied on the spectrum, and the depth structural reconstruction process was performed. This process was then repeated with the Gaussian window scanning through the whole spectral range, and the wavelengthdependent 3D structure was obtained. The reduced range of spectrum relaxed the axial resolution to be about $20 \mu \mathrm{m}$. The averaged spectra from the specimen were modelled by $I(k)=k^{2-D / 2}$. The value of $D$ was then obtained by calculating the power of the spectra.

Statistical analysis. Data are presented as mean \pm s.e.m., and $P$ values were determined using a Mann-Whitney test for single comparisons. For comparison of relative numbers of mice containing metastasis to various tissues, a Fisher's exact test was used to determine the $P$ value. Statistical analysis was performed using GraphPad Prism.

\section{References}

1. Griffith, O. L. \& Gray, J. W. 'Omic approaches to preventing or managing metastatic breast cancer. Breast Cancer Res. 13, 230 (2011).

2. Nagrath, S. et al. Isolation of rare circulating tumour cells in cancer patients by microchip technology. Nature 450, 1235-1239 (2007).

3. Stott, S. L. et al. Isolation of circulating tumor cells using a microvortexgenerating herringbone-chip. Proc. Natl Acad. Sci. USA 107, 18392-18397 (2010).

4. Yoon, H. J. et al. Sensitive capture of circulating tumour cells by functionalized graphene oxide nanosheets. Nat. Nanotechnol 8, 735-741 (2013).

5. Bednarz-Knoll, N., Alix-Panabieres, C. \& Pantel, K. Clinical relevance and biology of circulating tumor cells. Breast Cancer Res. 13, 228 (2011).

6. Lacroix, M. Significance, detection and markers of disseminated breast cancer cells. Endocr. Relat. Cancer 13, 1033-1067 (2006).

7. Paget, S. The distribution of secondary growths in cancer of the breast. 1889 . Cancer Metastasis Rev. 8, 98-101 (1989).

8. Kaplan, R. N. et al. VEGFR1-positive haematopoietic bone marrow progenitors initiate the pre-metastatic niche. Nature 438, 820-827 (2005).

9. Erler, J. T. et al. Hypoxia-induced lysyl oxidase is a critical mediator of bone marrow cell recruitment to form the premetastatic niche. Cancer Cell 15, 35-44 (2009).

10. Peinado, H., Lavotshkin, S. \& Lyden, D. The secreted factors responsible for pre-metastatic niche formation: old sayings and new thoughts. Semin. Cancer Biol. 21, 139-146 (2011). 
11. Sleeman, J. P. The metastatic niche and stromal progression. Cancer Metastasis Rev. 31, 429-440 (2012).

12. Qian, B. et al. A distinct macrophage population mediates metastatic breast cancer cell extravasation, establishment and growth. PLoS One 4, e6562 (2009).

13. Gabrilovich, D. I. \& Nagaraj, S. Myeloid-derived suppressor cells as regulators of the immune system. Nat. Rev. Immunol. 9, 162-174 (2009).

14. Hiratsuka, S., Watanabe, A., Aburatani, H. \& Maru, Y. Tumour-mediated upregulation of chemoattractants and recruitment of myeloid cells predetermines lung metastasis. Nat. Cell Biol. 8, 1369-1375 (2006)

15. Sceneay, J. et al. Primary tumor hypoxia recruits CD11b $+/$ Ly6Cmed/Ly6G + immune suppressor cells and compromises NK cell cytotoxicity in the premetastatic niche. Cancer Res. 72, 3906-3911 (2012).

16. Qian, B. Z. et al. CCL2 recruits inflammatory monocytes to facilitate breast-tumour metastasis. Nature 475, 222-225 (2011).

17. Lee, J. et al. Implantable microenvironments to attract hematopoietic stem/ cancer cells. Proc. Natl Acad. Sci. USA 109, 19638-19643 (2012).

18. Moreau, J. E. et al. Tissue-engineered bone serves as a target for metastasis of human breast cancer in a mouse model. Cancer Res. 67, 10304-10308 (2007).

19. Erez, N. \& Coussens, L. M. Leukocytes as paracrine regulators of metastasis and determinants of organ-specific colonization. Int. J. Cancer 128, 2536-2544 (2011).

20. Solinas, G., Marchesi, F., Garlanda, C., Mantovani, A. \& Allavena, P. Inflammation-mediated promotion of invasion and metastasis. Cancer Metastasis Rev. 29, 243-248 (2010).

21. Yoneda, T., Williams, P. J., Hiraga, T., Niewolna, M. \& Nishimura, R. A bone-seeking clone exhibits different biological properties from the MDAMB-231 parental human breast cancer cells and a brain-seeking clone in vivo and in vitro. J. Bone. Miner. Res. 16, 1486-1495 (2001).

22. Yi, J. \& Backman, V. Imaging a full set of optical scattering properties of biological tissue by inverse spectroscopic optical coherence tomography. Opt. Lett. 37, 4443-4445 (2012).

23. Fujimoto, J. G. Optical coherence tomography for ultrahigh resolution in vivo imaging. Nat. Biotechnol. 21, 1361-1367 (2003).

24. Huang, D. et al. Optical coherence tomography. Science 254, 1178-1181 (1991).

25. Yi, J. et al. Can OCT be sensitive to nanoscale structural alterations in biological tissue? Opt. Express. 21, 9043-9059 (2013).

26. Backman, V. \& Roy, H. K. Advances in biophotonics detection of field carcinogenesis for colon cancer risk stratification. J. Cancer 4, 251-261 (2013).

27. Nadiarnykh, O., LaComb, R. B., Brewer, M. A. \& Campagnola, P. J. Alterations of the extracellular matrix in ovarian cancer studied by second harmonic generation imaging microscopy. BMC Cancer 10, 94 (2010).

28. Radosevich, A. J. et al. Ultrastructural alterations in field carcinogenesis measured by enhanced backscattering spectroscopy. J. Biomed. Opt. 18, 97002 (2013).

29. Turzhitsky, V. et al. Investigating population risk factors of pancreatic cancer by evaluation of optical markers in the duodenal mucosa. Dis. Markers 25, 313-321 (2008).

30. Yi, J. et al. Spatially resolved optical and ultrastructural properties of colorectal and pancreatic field carcinogenesis observed by inverse spectroscopic optical coherence tomography. J. Biomed. Opt. 19, 36013 (2014).

31. Stypula-Cyrus, Y. et al. HDAC up-regulation in early colon field carcinogenesis is involved in cell tumorigenicity through regulation of chromatin structure. PLoS One 8, e64600 (2013).

32. Ko, C. Y. et al. The use of chemokine-releasing tissue engineering scaffolds in a model of inflammatory response-mediated melanoma cancer metastasis. Biomaterials 33, 876-885 (2012).

33. Davis, M. E. et al. Injectable self-assembling peptide nanofibers create intramyocardial microenvironments for endothelial cells. Circulation 111, 442-450 (2005)

34. Yu, J. et al. The effect of stromal cell-derived factor-1alpha/heparin coating of biodegradable vascular grafts on the recruitment of both endothelial and smooth muscle progenitor cells for accelerated regeneration. Biomaterials 33, 8062-8074 (2012)

35. Lim, W. H. et al. Stent coated with antibody against vascular endothelialcadherin captures endothelial progenitor cells, accelerates re-endothelialization, and reduces neointimal formation. Arterioscler. Thromb. Vasc. Biol. 31, 2798-2805 (2011)

36. Zhang, W. et al. VEGF and BMP-2 promote bone regeneration by facilitating bone marrow stem cell homing and differentiation. Eur. Cell Mater 27, 1-12 (2014).

37. Shi, C. et al. Stem-cell-capturing collagen scaffold promotes cardiac tissue regeneration. Biomaterials 32, 2508-2515 (2011).
38. Gower, R. M. et al. Modulation of leukocyte infiltration and phenotype in microporous tissue engineering scaffolds via vector induced IL-10 expression. Biomaterials 35, 2024-2031 (2014)

39. Graham, J. G. et al. PLG scaffold delivered antigen-specific regulatory $\mathrm{T}$ cells induce systemic tolerance in autoimmune diabetes. Tissue Eng. Part A 19, 1465-1475 (2013).

40. Jang, J. H., Rives, C. B. \& Shea, L. D. Plasmid delivery in vivo from porous tissue-engineering scaffolds: transgene expression and cellular transfection. Mol. Ther. 12, 475-483 (2005).

41. Yap, W. T. et al. Collagen IV-modified scaffolds improve islet survival and function and reduce time to euglycemia. Tissue Eng. Part A 19, 2361-2372 (2013).

42. Yu, S. et al. Tumor exosomes inhibit differentiation of bone marrow dendritic cells. J. Immunol. 178, 6867-6875 (2007)

43. Hiratsuka, S., Watanabe, A., Aburatani, H. \& Maru, Y. Tumor-mediated upregulation of chemoattractants and recruitment of myeloid cells predetermines lung metastasis. Nat. Cell Biol. 8, 1369-1375 (2006).

44. Talmadge, J. E. \& Gabrilovich, D. I. History of myeloid-derived suppressor cells. Nat. Rev. Cancer 13, 739-752 (2013).

45. Vakoc, B. J. et al. Three-dimensional microscopy of the tumor microenvironment in vivo using optical frequency domain imaging. Nat. Med 15, 1219-1223 (2009).

46. Rogers, J. D., Capoglu, I. R. \& Backman, V. Nonscalar elastic light scattering from continuous random media in the Born approximation. Opt. Lett. 34, 1891-1893 (2009).

47. Radosevich, A. J., Yi, J., Rogers, D. \& Backman, V. Structural length-scale sensitivities of reflectance measurements in continuous random media under the Born approximation. Opt. Lett. 37, 5220-5222 (2012).

48. Rogers, J. D., Radosevich, A. J., Yi, J. \& Backman, V. Modeling light scattering in tissue as continuous random media using a versatile refractive index correlation function. IEEE J. Sel. Top. Quantum Electron. 20, 7000514 (2014).

49. Yi, J. et al. Can OCT be sensitive to nanoscale structural alterations in biological tissue? Opt. Express 21, 9043-9059 (2013).

50. Dull, T. et al. A third-generation lentivirus vector with a conditional packaging system. J. Virol 72, 8463-8471 (1998).

\section{Acknowledgements}

231BR cells and support in development of the orthotopic tumour model were provided by V. Cryns, A. Mazar and the Northwestern University Developmental Therapeutics Core. G. Bushnell provided assistance with animal studies. Whole-animal imaging was performed at the Northwestern University Center for Advanced Molecular Imaging generously supported by NCI CCSG P30 CA060553. Flow cytometry work was supported by the Northwestern University Flow Cytometry Facility and a Cancer Center Support Grant (NCI CA060553). Confocal microscopy was performed at the Northwestern University Biological Imaging Facility on a Leica TCS SP5 laser scanning confocal microscope system purchased with funds from the NU Office for Research. This research was supported by the National Institutes of Health (R01CA173745) and the Northwestern H Foundation Cancer Research Award. The content is solely the responsibility of the authors and does not necessarily represent the official views of the $\mathrm{H}$ Foundation. B.A.A. is the recipient of a NSF Graduate Research Fellowship.

\section{Author contributions}

S.M.A., R.M.G, B.A.A., E.J.J. and S.S.R., performed tumour inoculations, implantation of biomaterials, and flow cytometry studies. A.G.G. cryosectioned tissues. S.M.A., E.J.J. and Y.R. fabricated scaffolds and performed H\&E staining. M.E.S. performed pathological analysis and scoring of tissue sections. S.M.A. performed immunohistochemistry and confocal imaging. B.A.A. and S.M.A. performed migration assays. J.Y. performed ISOCT imaging. S.M.A., J.Y., E.J.J. and S.L.T. Analysed data. V.B., J.S.J. and L.D.S. provided guidance and expertise. S.M.A., J.Y., V.B., J.S.J. and L.D.S. wrote and edited the manuscript.

\section{Additional information}

Supplementary Information accompanies this paper at http://www.nature.com/ naturecommunications

Competing financial interests: The authors declare no competing financial interests.

Reprints and permission information is available online at http://npg.nature.com/ reprintsandpermissions/

How to cite this article: Azarin, S. M. et al. In vivo capture and label-free detection of early metastatic cells. Nat. Commun. 6:8094 doi: 10.1038/ncomms9094 (2015). 